\title{
Encefalopatía hiperamonémica por ácido valproico, sin elevación de transaminasas: Reporte de Caso
}

\section{Hyperammonemic encephalopathy due to valproic acid, without elevation of transaminases: Case Report}

Isabel Eugenia Escobar-Toledo ${ }^{1}$, Marie Claire Berrouet-Mejía ${ }^{2}$

\section{RESUMEN}

Las encefalopatías tóxicas pueden ser causadas por una gran variedad de xenobióticos por mecanismos directos e indirectos simulando enfermedades metabólicas autoinmunes o neoplásicas. Por ende, requieren una anamnesis adecuada, un examen físico amplio y en cuanto a lo toxicológico tratar siempre de encontrar un

\begin{abstract}
Toxic encephalopathies can be caused by a wide variety of xenobiotics by direct and indirect mechanisms, simulating autoimmune or neoplastic metabolic diseases, therefore, they require an adequate anamnesis, a comprehensive physical examination and, in terms of toxicology, always try to find an epidemiological link.
\end{abstract}

Historial del artículo:

Fecha de recepción: 30/12/2020

Fecha de aprobación: 11/04/2021

1 Universidad de Antioquia, Clínica SOMA, SerVid-Clínica del Oriente. Médica y Cirujana, Especialista en Toxicología Clínica. Medellín, Colombia.

2 Universidad de Antioquia, Universidad CES, Clínica Hospital General de Medellín, Clínica SOMA. Grupo de investigación CES-HGM, Médica y Cirujana, Especialista en Toxicología Clínica, Magister en Epidemiología. Medellín, Colombia.

Correspondencia: Isabel Eugenia Escobar Toledo. Dirección: Carrera 75 \# 77AC-75 Int. 109 Medellín, Antioquia, Colombia. Correo Electrónico: isaescobartoledo@gmail.com

Como citar este artículo: Escobar-Toledo I, Berrouet MC. Encefalopatía Hiperamonémica por ácido valproico, sin elevación de transaminasas: Reporte de Caso. Revista de la Facultad de Ciencias de la Salud de la Universidad del Cauca. 2021;23(2):58-63. https:// doi.org/10.47373/rfcs.2021.v232.1823 
nexo epidemiológico. Dentro de las principales causas están psicofärmacos y drogas de abuso.

El reconocimiento temprano de una encefalopatía tóxica es fundamental entendiendo que llevará al retiro de la noxa, inicio de manejo disminuyendo complicaciones y mortalidad asociada. Se describe el caso de una paciente con ingesta de ácido valproico quien presentó una encefalopatía por este anticonvulsivante sin compromiso de enzimas hepáticas, con alteraciones en el electroencefalograma que revirtieron por completo con la suspensión delmedicamento.

La importancia de este caso está en reconocer al ácido valproico como una causa de encefalopatía.

Palabras clave: ácido valproico, Encefalopatía, Hiperamonemia, efectos adversos.

\section{INTRODUCCIÓN}

En el ańo 2016 en Estados Unidos se registraron 63488 casos de intoxicaciones por anticonvulsivantes, llegando a ser la séptima causa más frecuente de intoxicación por sustancias. En el ańo 2017, de acuerdo con las estadísticas de la American Association of Poison Control Centers (AAPCC), se registraron 65.941 casos de intoxicación por anticonvulsivantes; de ellos, 7.923 casos por ácido valproico, y dentro de ellos encontraron 81 casos con complicaciones mayores con 8 muertes registradas (1).

En Colombia en el ańo 2018, Estrada et al. publicaron una investigación sobre la epidemiología de las intoxicaciones en los servicios de urgencias en la ciudad de Medellín durante el periodo 2010-2013, encontrando que los anticonvulsivantes eran la causa en el $14.3 \%$ de los casos $(2,3)$.

El ácido valproico es un medicamento con una gran variedad de indicaciones, algunas como: estabilizador del ánimo, profilaxis de migrańa y anticonvulsivante. Considerando que los pacientes que consultan a urgencias por intoxicaciones o efectos adversos asociados al ácido valproico no desarrollan necesariamente un toxídrome específico, se hace necesario conocer aspectos cinéticos, dinámicos, manifestaciones clínicas, que permitan establecer un nexo epidemiológico, hacer un abordaje diagnóstico y terapéutico adecuado optimizando el uso de ayudas diagnósticas (4-6).
Among the main causes are psychotropic drugs and drugs of abuse.

The early recognition of a toxic encephalopathy is essential, understanding that it will lead to the withdrawal of noxa, initiation of management, reducing complications and associated mortality. We describe the case of a patient with valproic acid treatment who develops encephalopathy due to this anticonvulsant without compromising liver enzymes, the importance of this case is in recognizing valproic acid as a cause of encephalopathy.

Key words: Valproic Acid, encephalopathy, hyperammonemia, adverse effects

El ácido valproico [ácido n-dipropilacético] químicamente puede ser clasificado como un ácido carboxílico; las dosis terapéuticas van desde $15 \mathrm{mg} / \mathrm{kg} /$ día hasta un máximo de $60 \mathrm{mg} / \mathrm{kg} /$ día y sus concentraciones plasmáticas terapéuticas oscilan entre 50 y $150 \mu \mathrm{g} / \mathrm{mL}$. Viene en presentaciones parenterales y orales (de liberación inmediata y prolongada). Luego de su ingesta, su biodisponibilidad es superior al $80 \%$, los alimentos pueden retrasar la absorción de las presentaciones orales. El volumen de distribución de este medicamento es de $0.14-0.2 \mathrm{~L} / \mathrm{kg}$ y la unión a proteínas puede ir entre 74 - 93\%, aunque esta unión puede disminuir en poblaciones especiales como los neonatos, ancianos, pacientes con disfunción hepática o renal (5-7).

El ácido valproico se metaboliza en el hígado con una cinética de eliminación de primer orden, principalmente a través de mecanismos de conjugación (30-50\%), beta-oxidación mitocondrial (40\%) y omega-oxidación microsomal $(15-20 \%)(4,5,8)$.

El ácido valproico se ha asociado a diferentes efectos adversos en el sistema nervioso central, dentro de los que se encuentra la encefalopatía. A continuación, se describe el caso de una paciente que desarrolló una encefalopatía hiperamonémica, complicación que se ha descrito frecuentemente asociada a interacciones con otros medicamentos y alteración de pruebas hepáticas, no obstante, el hallazgo de encefalopatía y el nexo de uso de ácido valproico, deben hacer sospechar esta condición. 
Este caso reporta una paciente con encefalopatía por ácido valproico, potenciada posiblemente por la neurotoxicidad de cocaína, sin compromiso de pruebas hepáticas y ese es el aspecto novedoso por el cual se reporta este caso (9).

\section{REPORTE DE CASO}

Paciente femenina de 25 ańos, con antecedente de epilepsia desde los 12 ańos, caracterizada por crisis dialépticas, en tratamiento desde hace 5 ańos con ácido valproico $250 \mathrm{mg}$ cada 12 horas y Fenobarbital 200mg día. Consulta a un centro de mediana complejidad por un cuadro de tres días de evolución de varios episodios convulsivos tónicos generalizados, la presencia de vómitos y deterioro en el estado de alerta. Dentro de sus antecedentes estaba el consumo de cocaína y de inhalantes tipo sacol desde hace más de tres ańos, con un último consumo de inhalantes hace un mes y de cocaína un día antes de comenzar con los episodios convulsivos.

Al ingreso al servicio de urgencias estaba estable hemodinámicamente, con una presión arterial de 130/80 $\mathrm{mmHg}$, una frecuencia cardiaca de 85 latidos por minuto, frecuencia respiratoria de 18 por minuto y una Escala de Coma de Glasgow de 9/15. Presentó episodios convulsivos durante las primeras 48 horas por lo cual recibió manejo como un Estado Epiléptico con Diazepam $10 \mathrm{mg}$ por vía intravenosa, Midazolam $30 \mathrm{mg}$ intravenoso, ácido valproico $500 \mathrm{mg}$ día vía oral, Fenitoína 300 mg vía oral y Fenobarbital 200 mg vía oral.

Dada la persistencia de episodios convulsivos, fue remitida a un hospital de alta complejidad para valoración y manejo integral por Neurología Clínica. Al ingreso recibió tratamiento con Midazolam en bolos y ácido valproico; se realizó Tomografía Axial Computada (TAC) de cráneo simple y posteriormente punción lumbar, para descartar lesiones estructurales y posible compromiso infeccioso (Tabla 1).

Por la persistencia en el compromiso del estado de alerta es trasladada a Unidad de Cuidados Especiales donde se solicita electroencefalograma (EEG) de 48 horas; el estudio de las primeras 12 horas reportó Actividad epileptiforme bifrontal, inicial, independiente y sincrónica; sin evidencia de nueva actividad epileptiforme y trazado de fondo encefalopático con enlentecimiento Delta difuso, con variante de $14-6 \mathrm{~Hz}$ en cuadrantes posteriores.

Por evolución neurológica con pobre respuesta a estímulos y sin reflejos protectores de la vía aérea, se aseguró la vía respiratoria y fue trasladada a la Unidad de Cuidado Intensivo (UCI). Se solicitó valoración por Toxicología Clínica, quien considerando la alteración neurológica persistente y los hallazgos del EEG descritos en encefalopatía medicamentosa, se considera la posibilidad de una encefalopatía por ácido valproico.

Solicitaron niveles de ácido valproico y Fenobarbital los cuales se encontraban dentro del rango terapéutico, prueba de tóxicos en orina para evidenciar el último consumo y probables coingestas, con reporte positivo para cocaína, Benzodiacepinas y Barbitúricos. Niveles de Amonio elevados, por lo cual se retiró el ácido valproico y se inició manejo con Lactulosa 30 gramos tres veces al día por tres días, en la Tabla 1 . Se presentan los resultados de los paraclínicos.

Posterior a la suspensión del medicamento, el reporte electroencefalográfico evidenció mejoría en el enlentecimiento Delta difuso, así como en la organización y reactividad respecto a registros previos. Los niveles de Amonio disminuyeron rápidamente al valor normal y la paciente presentó mejoría progresiva del estado neurológico, logrando retirar el soporte ventilatorio, sin complicaciones, sin nuevos eventos convulsivos y sin hubo eventos adversos asociados al manejo.

La paciente confirmó la exposición a cocaína previo a la aparición del cuadro convulsivo; al estar asintomática se realizó intervención por Toxicología Clínica y se indicó tratamiento de rehabilitación multidisciplinario para adicciones, el cual fue aceptado por la paciente. Fue dada de alta sin secuelas neurológicas con formula ambulatoria de Levetiracetam $1000 \mathrm{mg}$ cada 8 horas para el manejo de su epilepsia. Durante el abordaje y seguimiento de la paciente no se tuvo ninguna dificultad para el diagnóstico.

\section{DISCUSIÓN}

Presentamos el caso de una paciente con encefalopatía hiperamonémica sin alteración de transaminasas, en el contexto de tratamiento crónico con ácido valproico en dosis terapéuticas y sin niveles séricos elevados del fármaco a pesar de tratamiento concomitante de larga evolución con Fenobarbital. La neurotoxicidad es influenciada por diferentes variables, dentro de las cuales vale la pena resaltar las propiedades físicas y químicas de los xenobióticos, así como las características propias del individuo, como la edad y condiciones como diabetes, falla renal, compromiso hepático que pueden favorecer la acumulación de sustancias, alterando el sistema nervioso central (SNC) (10). 
Tabla 1. Resultados de paraclínicos

\begin{tabular}{|c|c|c|c|c|c|c|c|}
\hline Paraclínicos & $09 / 05 / 20$ & $10 / 05 / 20$ & $11 / 05 / 20$ & $12 / 05 / 20$ & $13 / 05 / 20$ & $14 / 05 / 20$ & $15 / 05 / 20$ \\
\hline $\begin{array}{l}\text { Aspartato } \\
\text { aminotransferasa mg/dl }\end{array}$ & & 21 & & 16 & & 49 & \\
\hline $\begin{array}{l}\text { Alanino } \\
\text { aminotransferasa mg/dl }\end{array}$ & & 10 & & 13 & & 40 & \\
\hline $\begin{array}{l}\text { Fosfatasa alcalina } \\
\mathrm{mg} / \mathrm{dl}\end{array}$ & & 81 & & 88 & & 94 & \\
\hline $\begin{array}{l}\text { Bilirrubina total } \\
\mathrm{mg} / \mathrm{dl}\end{array}$ & & 0.96 & & & & 0.41 & \\
\hline $\begin{array}{l}\text { Bilirrubina directa } \\
\mathrm{mg} / \mathrm{dl}\end{array}$ & & 0.26 & & & & 0.14 & \\
\hline $\begin{array}{l}\text { Bilirrubina indirecta } \\
\mathrm{mg} / \mathrm{dl}\end{array}$ & & 0.7 & & & & 0.27 & \\
\hline $\begin{array}{l}\text { Niveles Ácido } \\
\text { Valproico mcg/ml }\end{array}$ & & & & 2 & & & \\
\hline $\begin{array}{l}\text { Niveles } \\
\text { Fenobarbital }\end{array}$ & & & & 49 & & & \\
\hline Amonio & & & & 287 & & 60 & \\
\hline $\mathrm{VIH}$ & Negativo & & & & & & \\
\hline VDRL & $\begin{array}{l}\text { No } \\
\text { reactivo }\end{array}$ & & & & & & \\
\hline Lactato $\mathrm{mmol} / \mathrm{L}$ & & 1 & 0.82 & & & & \\
\hline Creatinina mg/dl & & 0.57 & & 0.51 & & 0.38 & \\
\hline Tóxicos en orina & & & & $\begin{array}{c}\text { Cocaína } \\
\text { Benzodiace- } \\
\text { pinas } \\
\text { Barbitúricos }\end{array}$ & & & \\
\hline
\end{tabular}

La palabra encefalopatía hace alusión a un síndrome de disfunción cerebral y su característica fundamental es la alteración de la conciencia. Las encefalopatías tóxicas explican hasta el 30\% de las causas de encefalopatía.

El estado de conciencia está condicionado por la integridad y la relación entre dos estructuras: el sistema reticular activador ascendente (SARA) y la corteza cerebral.

Dentro de los diferentes aspectos a considerar en la fisiopatología de las encefalopatías tóxicas están: relación dosis-respuesta, riesgos hepáticos, cardiovasculares o renales que refuercen el concepto de una etiología tóxica; en este caso, además del cuadro clínico, se encontró el amonio elevado, pero llamó la atención la ausencia de compromiso hepático como se encuentra en otros casos reportados en la literatura $(9,12)$.

En el caso que reportamos, la encefalopatía podía ser explicada por ácido valproico y cocaína, ya que este alcaloide puede producir dańo en el sistema nervioso. Las principales complicaciones de la ingesta aguda de cocaína son secundarias al vasoespasmo e incluyen convulsiones, déficit isquémico transitorio y síndrome neurovascular isquémico y hemorrágico. En este caso, por el tiempo transcurrido y la falta de síntomas adrenérgicos, es más plausible la asociación con ácido valproico, entendiendo una exposición más prolongada, el aumento de dosis 
reciente y la falta de focalización, que se esperaría más en cocaína $(13,14)$.

La hiperamonemia asociada al ácido valproico se define típicamente como una concentración plasmática de amonio superior a $80 \mathrm{mcg} / \mathrm{dL}(47 \mu \mathrm{mol} / \mathrm{L})$. Con respecto a la fisiopatología, el ácido valproico se une en el hepatocito a la Coenzima-A (CoA) en el citoplasma, por medio de la enzima Acil-transferasa I y subsecuentemente, el fármaco es transferido a la carnitina, lo que permite el ingreso a la mitocondria y allí nuevamente se trasfiere a la CoA, por la Aciltransferasa II, iniciando el proceso de $\beta$-oxidación mitocondrial generando diversos metabolitos, los cuales secuestran a la CoA, disminuyendo la formación del N-AcetilGlutamato, un cofactor obligatorio para la Carbamoil-Fosfato Sintetasa I (CPS I), enzima que es responsable de la incorporación del amonio en el ciclo de la úrea (13).

La prevalencia de hiperamonemia inducida por valproato en la población general de pacientes que toman este medicamento no está bien establecida, pero es probable que sea mayor en aquellos que reciben atención en los centros médicos. Los estudios transversales han descrito una prevalencia de $16 \%(16,17)$.

En este caso, al igual que lo reportado por Cano et al. en el 2012, se encontró la presencia de otros medicamentos que disminuyen la excreción normal del amonio, como el fenobarbital que inhibe la síntesis de glutamina sintasa cerebral, enzima que metaboliza el glutamato y amonio a glutamina, explicando la encefalopatía; sin embargo, un hallazgo que llama la atención, es que nunca elevó transaminasas a pesar de los factores de riesgo que tenía como la exposición a cocaína y la polimedicación 180 (9,17,18).

\section{CONCLUSIONES}

En pacientes con alteraciones de la conciencia y que reciben tratamiento con ácido valproico es importante solicitar niveles de amonio independiente de la presencia de otras alteraciones. En caso de tener elevados estos niveles el tratamiento de elección en la encefalopatía hiperamonémica es suspender el anticonvulsivante; si esta medida es insuficiente, requerirá manejo con carnitina 50 a $100 \mathrm{mg} / \mathrm{kg}$ descrito como el tratamiento para la intoxicación aguda por ácido valproico, uno de los diagnósticos diferenciales (19). La encefalopatía hiperamonémica es una reacción adversa grave que puede estar subdiagnósticada, y comprometer la vida de los pacientes.
Este caso nos invita a estar atentos ante las diferentes manifestaciones de neurotoxicidad en pacientes que reciben tratamiento con psicofármacos y son consumidores de sustancias psicoactivas.

\section{REFERENCIAS}

1. Gummin DD, Mowry JB, Spyker DA, Brooks DE, Fraser MO, Banner W. 2016 Annual Report of the American Association of Poison Control Centers' National Poison Data System (NPDS): 34th Annual Report. Clin Toxicol (Phila). 2017; 55(10):1072-1252. Doi: 10.1080/15563650.2017.1388087

2. Estrada AF, Berrouet MC, Zuluaga M, Ortiz A. Epidemiología de las intoxicaciones agudas en los servicios de urgencias hospitalarias. Medellín, Colombia. Rev. Toxicol. 2018; 35: 9-123.

3. Berrouet MC, Zuluaga M, Estrada AF, Diaz J, Gonzalez S. Utilidad de la hemodiálisis en intoxicación por anticonvulsivantes: reporte de dos casos. Rev. Toxicol. 2019; 36:138-141

4. Peńa A LM, Arroyave H CL, Aristizábal H JJ, Gómez C UE. Fundamentos de medicina: Toxicología clínica. Cap. 29. Intoxicación por anticonvulsivantes: Carbamazepina, Fenitoína, Ácido valproico. 1.Ş ed. Medellín: Corporación para Investigaciones Biológicas; 2010.

5. Goodman L, Gilman A. The pharmacological basis of therapeutics. McGraw Hill Publishing Co. Inc. New York, 2012,12th edition, 591-593.

6. Kostrouchová M, Kostrouch Z, Kostrouchová M. Valproic acid, a molecular lead to multiple regulatory pathways. Folia Biol (Praha). 2007; 53(2):37-49.

7. Sztajnkrycer MD. Valproic acid toxicity: overview and management. J Toxicol Clin Toxicol. 2002; 40(6):789801. Doi: $10.1081 /$ clt-120014645

8. Kearney, Thomas E. Chapter 153. Valproic Acid. Poisoning \& Drug Overdose, 6e Ed. Kent R. Olson. McGraw Hill, 2012, Disponible en: https://accessmedicine.mhmedical.com/ content. aspx?bookid=391\&sectionid=42069967.

9. Berrouet MC, Chica V. Anticonvulsivantes. En: Guías para el manejo de emergencias toxicológicas. Ministerio de Protección Social. Organización Panamericana de la salud. Convenio 344 de 2016. Bogotá, junio 30 de 2017.

10. Cano A, Hernández 0, González C, Amariles P. Encefalopatía hiperamonémica asociada a ácido valproico por posible sinergismo con fenobarbital y topiramato: comunicación de tres casos. Farmacia Hospitalaria. 2012; 36(5), 441-442. Doi: 10.1016/j.farma.20.09.005 
11. Rao M. Neurological prinicples In. Goldfrank LR, Flomenbaum NE, Lewin NA, Howland MA, Hoffman RS, Nelson LS. Goldfrank's Toxicologic Emergencies. McGrawHill 11 th Edition. New York, 2017. Disponible en: https:// accesspharmacy.mhmedical.com/book.aspx?bookID=2569

12. Edlow JA, Rabinstein A, Traub SJ, et al. Diagnosis of reversible causes of coma. Lancet. 2014; 384(9959):2064-76. Doi: 10.1016/S0140-6736(13)62184-4.

13. Berrouet MC, Pino-Ramos NE., Castro-Botero M., GómezCalzada UE. Alteración del estado de conciencia en el paciente intoxicado. Medicina UPB. 2017; 36(1):71-79. https://Doi.org/10.18566/medupb.v36n1.a09.

14. Rupasinghe J, Jasinarachchi M. Progressive encephalopathy with cerebral oedema and infarctions associated with valproate and diazepam overdose. J Clin Neurosci. 20; 18:710.

15. Rico-Mesa JS, Rico-Mesa MA, Berrouet MC. Ischemic stroke related to acute consumption of cocaine [Internet]. CES Med. 2017; 31(2):207-14. Disponible en: https://revistas. ces.edu.co:443/index.php/medicina/article/view/3879

16. Chicharro AV, de Marinis AJ, Kanner AM. The measurement of ammonia blood levels in patients taking valproic acid: looking for problems where they do not exist?. Epilepsy Behav. 2007; 11(3):361-6. Doi: 10.1016/j.yebeh.2007.06.015.

17. Carr Russell B, Shrewsbury K. Hyperammonemia due to valproic acid in the psychiatric setting. Am J Psychiatry. 2007; 164(7):1020-7. Doi: 10.1176/ ajp.2007.164.7.1020.

18. Hamer HM, Knake S, Schomburg U, Rosenow F. Valproate induced hyperammonemic encephalopathy in the presence of topiramate. Neurology. 2000; 54(1):230-2. Doi: 10.1212/wnl.54.1.230.

19. Glatstein M, Bonifacio Rino P, de Pinho S, Scolnik D, Pivko-Levi D, Hoyte C. Levocarnitine for the Treatment of Valproic Acid-Induced Hyperammonemic Encephalopathy in Children: The Experience of a Large, Tertiary Care Pediatric Hospital and a Poison Center. Am J Ther. 2019; 26(3):344-349. Doi: 10.1097/ MJT.0000000000000706. 